\title{
Normal pressure hydrocephalus
}

\author{
How often does the diagnosis hold water?
}

[

Bryan T. Klassen, MD

J. Eric Ahlskog, PhD, MD

Address correspondence and reprint requests to Dr. Bryan T. Klassen, Department of Neurology, Mayo Clinic Rochester, 200 First Street SW, Rochester, MN 55905

klassen.bryan@mayo.edu

\section{ABSTRACT}

Objective: To determine our community's incidence of clinically suspected normal pressure hydrocephalus (NPH), the rate of shunting for NPH, and short- and long-term outcomes of shunting.

Methods: A retrospective query of the Mayo Clinic medical records linkage system was conducted to identify residents of Olmsted County, Minnesota, undergoing an invasive diagnostic procedure for evaluation of suspected NPH from 1995 through 2003. Among patients with shunts, earlyand long-term outcomes were determined via a review of available medical records.

Results: Forty-one patients underwent an invasive diagnostic procedure for evaluation of suspected NPH; 13 ultimately received shunts, representing an incidence of 1.19/100,000/year. The incidence of sustained definite improvements at 3 years after shunting was only $0.36 /$ $100,000 / y e a r$. Definite gait improvement was documented in $75 \%$ at 3-6 months after shunt placement, although it dropped to $50 \%$ at 1 year and to $33 \%$ at 3 years. Only 1 of 8 patients with cognitive impairment and 1 of 6 patients with urinary incontinence had definite improvement in these symptoms at 3 years. No patient with moderate to severe postural instability experienced sustained definite improvement in any symptom. Complications occurred in 33\% of patients including one perioperative death. Additional or alternative neurologic diagnoses later surfaced in 5 of 12 patients.

Conclusions: In this community, NPH is relatively rare with an incidence approximating that of progressive supranuclear palsy in this population. Whereas early gait improvement was common, only one-third of patients enjoyed continued improvement by 3 years; cognition or urinary incontinence was even less responsive long term. Baseline postural instability predicted poor outcome. Clinicians should balance potential benefits of shunting against the known risks. Neurology ${ }^{\circledR} 2011$; 77:1119-1125

\section{GLOSSARY}

H-ICDA = International Classification of Diseases, Adapted Code for Hospitals; NPH = normal pressure hydrocephalus; OMC = Olmsted County Medical Center; $\mathbf{P S P}=$ progressive supranuclear palsy .

The patient presenting with gait disturbance, cognitive decline, or urinary incontinence represents a common clinical dilemma for the practicing neurologist. Although these symptoms are suggestive of normal pressure hydrocephalus $(\mathrm{NPH}),{ }^{1,2}$ they are not specific to the diagnosis and commonly occur in neurodegenerative conditions or nonspecifically in advanced age. Ventriculomegaly, the radiologic hallmark of NPH, frequently accompanies both neurodegenerative disease and normal aging; in practice, it is difficult to determine whether ventricular enlargement results from hydrocephalus or ex vacuo change. The diagnostic uncertainty in these patients is particularly problematic, given the invasive nature of treatment for NPH. Although published guidelines generally support the role of CSF removal trials in assisting with the diagnosis, ${ }^{3}$ neither this nor any other test reliably assures sustained benefit from shunting. In recent years, we have noted an increase in the number of patients referred to our movement disorders practice for evaluation of possible NPH, perhaps driven partly by increased public awareness from media coverage and device manufacturer-funded advertising. It was our clinical

From the Department of Neurology, Mayo Clinic Rochester, Rochester, MN.

Study funding: Supported by the National Institute on Aging (grant R01 AG034676).

Disclosure: Author disclosures are provided at the end of the article. 
impression that many of these patients were ultimately not thought to have NPH, often because alternate diagnoses better explain their symptoms. This raised the question as to the true frequency of NPH and, furthermore, the short- and long-term benefit from shunting.

At Mayo Clinic in Rochester, the previously reported early experience with NPH included a generally favorable early response to shunting among appropriately selected patients. ${ }^{2}$ As a result, Mayo Clinic neurologists, neurosurgeons, and neuroradiologists maintain a high level of vigilance for the clinical and radiologic signs of NPH. Given our institution's openness to this diagnosis and its position as our county's sole neurosurgical center, we thought that the experience with $\mathrm{NPH}$ in our Olmsted County population should have general validity. Thus, in this community-based study, we determined the following over a 9-year period (1995-2003): 1) the incidence of clinically suspected NPH, for which the clinician was sufficiently suspicious of the diagnosis to proceed with an invasive procedure (usually lumbar CSF removal); 2) the incidence of shunting for NPH; 3) the outcomes (both short-term and longterm) among patients with shunts in Olmsted County, MN.

METHODS Standard protocol approvals, registrations, and patient consents. Our retrospective chart review was approved by the Mayo Clinic Institutional Review Board, and written informed consent for participation in research was on file for all patients included.

Study population and community referral patterns. Olmsted County, located in southeastern Minnesota, has a population of 124,277, according to the year 2000 national census. Primary healthcare services are delivered through the Mayo Clinic, affiliated clinics within Mayo Health Systems, Olmsted County Medical Center (OMC), and private providers. During the years covered by the study, neurologic consultations were only available through the Mayo Clinic Department of Neurology and through a single neurologist at OMC. The Mayo Clinic remains the county's sole neurosurgical center, and, for this reason, the OMC neurologist chose to refer all patients with suspected $\mathrm{NPH}$ to the Mayo Clinic before their undergoing a high-volume lumbar puncture or other invasive diagnostic procedure.

Case definition. Cases of clinically suspected NPH were operationally defined as those in which a patient underwent an invasive diagnostic or therapeutic procedure for the evaluation or treatment of suspected NPH; this almost always included a highvolume lumbar puncture, although lumbar cisternography, continuous CSF drainage trials, or ventriculoperitoneal shunting was also allowed within our definition. At our institution, only neurologists or neurosurgeons may order these procedures, ensuring that patients falling within our definition would have been carefully screened for both NPH and competing neurologic diagnoses. Subjects with a history of congenital hydrocephalus, CNS hemorrhage, venous sinus thrombosis, CNS infection, or an obstructing lesion were excluded from analysis.

Patient ascertainment. The Mayo Clinic medical records linkage system was retrospectively queried to identify Olmsted County residents who had a clinical encounter between 1995 and 2003 designated with the International Classification of Diseases, Adapted Code for Hospitals (H-ICDA) diagnostic codes for NPH, hydrocephalus NOS, communicating hydrocephalus, or occult hydrocephalus. To assure capture of all patients in whom NPH was considered, a computerized search of the text of all clinical notes covering all county residents during this time frame was also performed using the terms normal pressure hydrocephalus or communicating hydrocephalus. The search strategy included primary care notes, allowing capture of patients primarily managed at Mayo Clinic, who had undergone surgical intervention outside the county (one such patient was, indeed, included). The ascertainment period was chosen such that all relevant clinical notes were accessible for the text-based search and adequate follow-up time was available at the time of analysis. The complete medical history of each patient identified was reviewed to determine whether a high-volume lumbar puncture or other invasive procedure was performed.

Data collection and determination of outcome. For identified cases, information collected included patient demographics, symptom duration, the presence or absence of each element of the NPH triad, the degree of postural instability, and the radiologist's impression of neuroimaging studies. For patients undergoing shunting, early (3-6 months) and late (3 years) outcomes were retrospectively determined by a comprehensive chart review to the last available follow-up. Based on the documented impressions of the treating neurologist, the patient, and the patient's family, outcomes were rated for each element of the clinical triad as follows: 0 , no improvement beyond presurgical baseline; \pm , equivocal improvement; + , definite improvement but significant residual symptoms; or ++ , normalization or near-normalization. In the case of a decline in patient status, the time to decline was determined using the date of the clinical assessment at which the decline was first documented. Complications of the shunting procedure were tabulated. Additional neurologic diagnoses made during follow-up were noted, as were the results of neuropathologic examination when available.

Determination of incidence rates. Population denominators used in the calculation of incidence rates were determined by a linear interpolation of the decennial census data with a $1.9 \%$ projected growth rate after 2000 . Average annual incidence rates (incidence per 100,000 person-years) were calculated for both the entire population and for the population aged 50-99 years. Because the study covered the entire target population, no sampling was involved, and, therefore, no statistical testing was applied during interpretation of the data.

RESULTS The H-ICDA database query returned 247 patients; 164 additional patients were discovered using the text-based search. From the 411 total records reviewed, 41 patients meeting the inclusion/ exclusion criteria were identified; the remainder were patients with congenital or secondary hydrocephalus 
or patients in whom the treating clinician's note explicitly dismissed NPH as a likelihood (such cases were flagged by the text-based search). All patients in whom NPH was felt to be the likely diagnosis were referred for invasive diagnostic testing. These $41 \mathrm{pa}-$ tients correspond to an incidence rate for clinically suspected NPH of 3.74/100,000/year for the total population and 15.20/100,000/year for the population older than 50 years.

Among the 41 patients with suspected NPH, all had gait disturbance, 30 had cognitive impairment, and 14 had urinary incontinence. Twenty of the 41 presented with 2 key symptoms, and 12 presented with all 3 elements of the NPH triad. Nineteen were found to have moderate to severe postural instability, defined by a positive pull test. Neuroimaging results were available for the 40 patients undergoing the invasive procedure at Mayo Clinic and, in all patients, documented ventriculomegaly.

Our practice has been to offer shunt placement only to patients with objective gait improvement after a trial of CSF removal. Of the 38 patients undergoing high-volume lumbar puncture, 14 experienced gait improvement, and 11 subsequently underwent ventriculoperitoneal shunting. Among the 3 of the 14 patients who did not undergo shunting, one had a history of carbon monoxide exposure and was later diagnosed with levodopa-responsive parkinsonism, another had a history of stroke, failed to appear for the neurosurgical evaluation, and was later diagnosed with multi-infarct dementia, and the third had a history of schizophrenia and declined neurosurgical intervention. Among the 41 patients with suspected $\mathrm{NPH}, 2$ did not undergo a trial of CSF removal but underwent other invasive procedures. One underwent lumbar cisternography with results not consistent with the diagnosis; hence, this patient did not undergo shunting. The other (case 1) was taken directly for ventriculoperitoneal shunting on the basis of gait dysfunction, urinary incontinence, and imaging studies thought to be highly typical of NPH. Finally, one patient underwent ventriculoperitoneal shunting at the regional Veterans Affairs Medical Center, and outcome data for that patient were unavailable; this patient is included in the epidemiology but not outcomes sections of this study.

Of the 41 patients in whom suspicion for $\mathrm{NPH}$ was sufficient to justify an invasive diagnostic or therapeutic procedure, 13 (32\%) ultimately underwent ventriculoperitoneal shunting (table). This corresponds to a shunted NPH incidence rate of $1.19 /$ $100,000 /$ year for the total population and 4.82/ $100,000 /$ year for those older than 50 years. The mean age at the time of shunting was 78.5 years (range 66-85 years). The gender distribution was nearly equal with 6 (46\%) women and 7 (54\%) men having undergone shunting. The average duration of symptoms before shunting was 2.4 years (range $0.4-$ 7.0 years). All 13 patients undergoing shunting had gait disturbance; 8 also had cognitive dysfunction and 6 had urinary incontinence. Six patients presented with 2 of the 3 symptoms, but only 4 patients presented with all elements of the classic NPH triad. Postural instability was absent in 4 patients, mild in 3 patients, and moderate to severe in 6 patients.

Outcome data were available for all 12 patients who underwent shunting at the Mayo Clinic. At 3-6 months postprocedure, 9 of these $(75 \%)$ demonstrated definite gait improvement; 2 did not respond to shunting, and 1 died in the perioperative period. By 1 year, only half (6 of 12) experienced definite gait improvement and by the 3-year endpoint only one-third (4 of 12) maintained definite improvement, whereas 5 of the original 9 responders (45\%) had a decline in gait response. One of these (case 7) was found to have orthostatic myoclonus as a possible contributor to gait decline, although no new causes of gait dysfunction were present in the remaining 4 . None of the 6 patients with moderate to severe postural instability experienced definite improvement in gait at the last follow-up. In cases 6, 9, and 10, the length of follow-up was less than 3 years; however, in each of these patients the benefit from shunting had already waned before the time of last follow-up, and shunt dysfunction or unrelated causes of gait dysfunction were considered and ruled out using appropriate studies. It was assumed that spontaneous improvement would not subsequently occur, and the clinical status at the last follow-up was carried forward for these 3 patients. Of the 8 patients with cognitive impairment, 4 (50\%) demonstrated definite early cognitive improvement after shunting, but only 1 (13\%) sustained improvement at 3 years. Similarly, of the 6 patients with urinary incontinence, 3 (50\%) demonstrated definite early improvement in urinary symptoms after shunting, but only 1 (16\%) sustained improvement at 3 years. If the patient who died perioperatively is removed from the denominator, favorable outcomes improve in the short term to 9 of 11 (82\%) for gait and 4 of 7 (57\%) for cognition and in the long term to 4 of 11 (36\%) for gait and 1 of 7 (14\%) for cognition. No new causes of cognitive decline or incontinence were found during life for any of the patients whose initial response to these symptoms waned.

No patient had definite improvement in cognition or urinary incontinence without concomitant definite improvement in gait at the same time point; therefore, gait outcomes were used to gauge the overall success of shunting. There was no clear relation- 


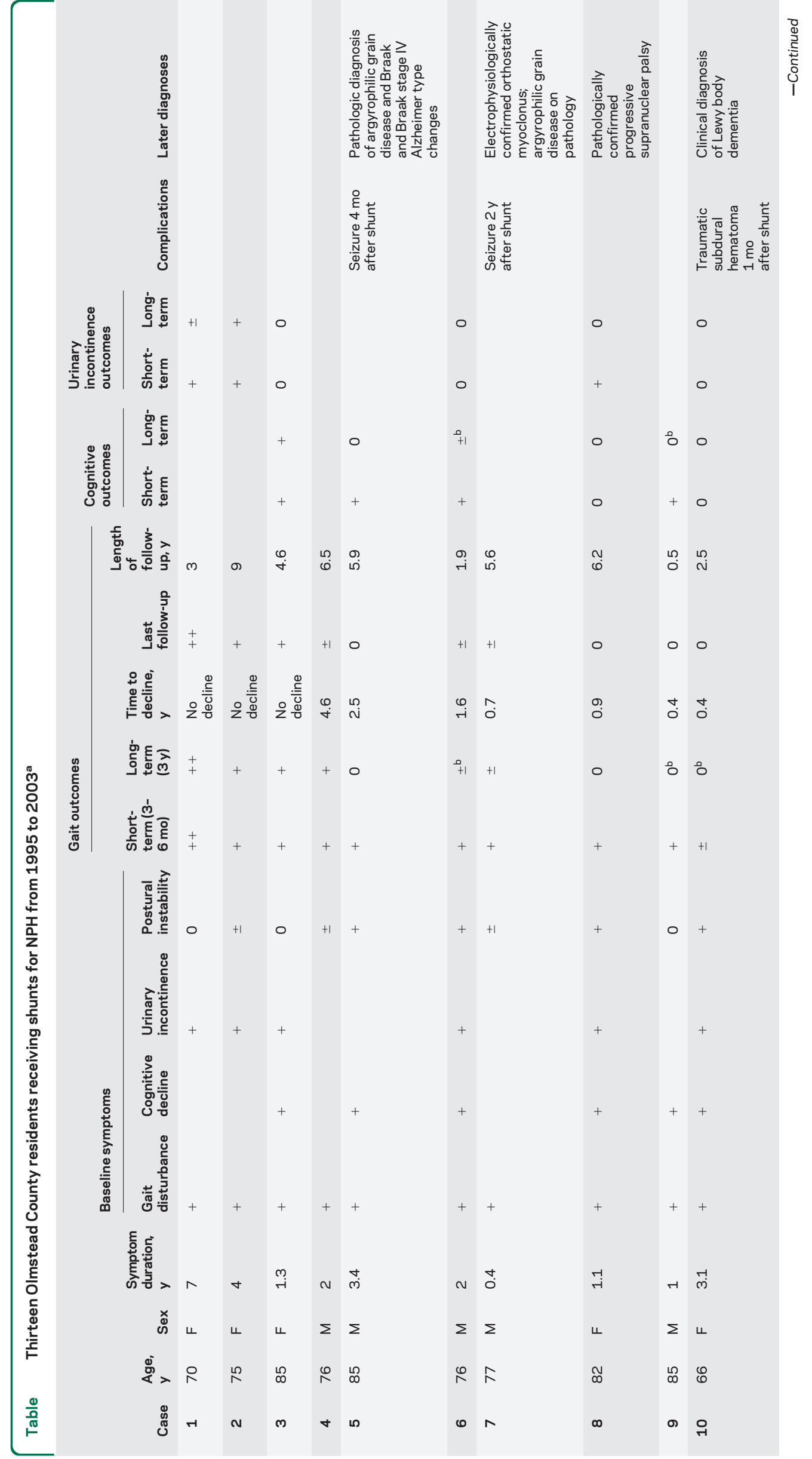




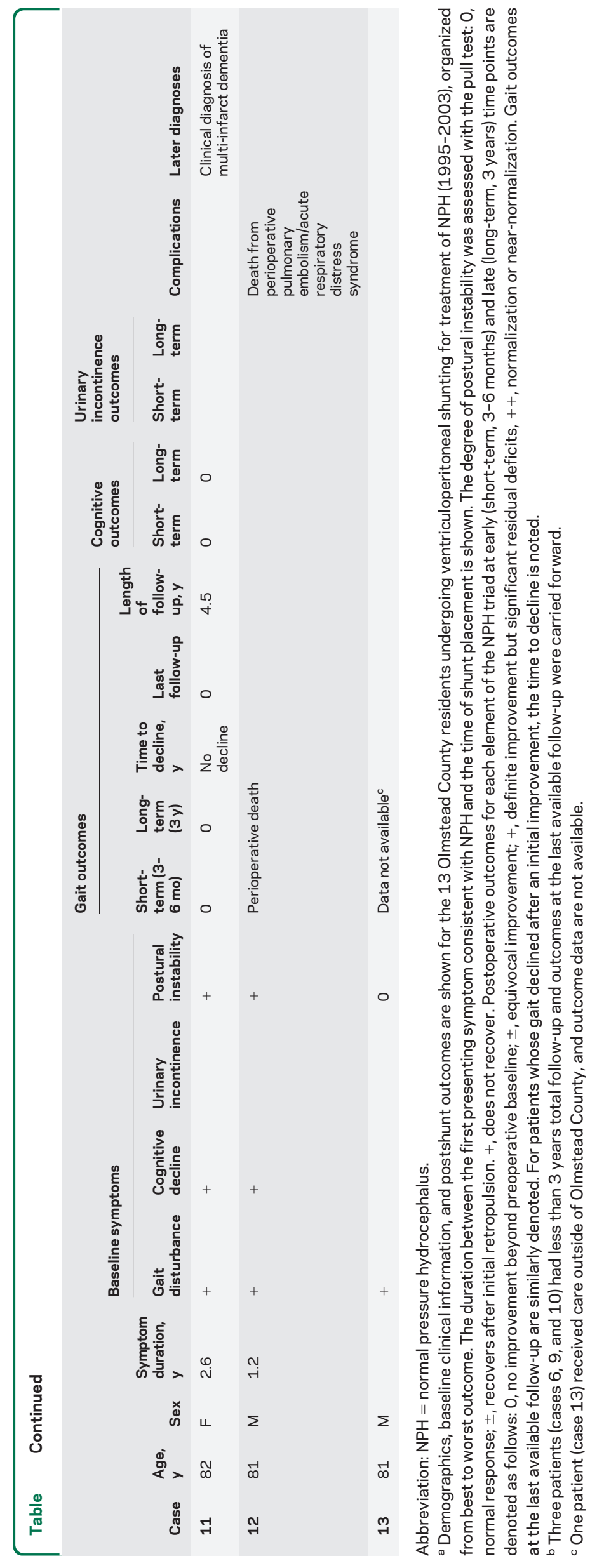

ship between symptom duration and shunt response; in fact, the patient with the longest symptom duration (7 years) was among the best responders. The incidence rate for a sustained definite improvement in any symptom at 3 years after shunting was $0.36 /$ $100,000 /$ year for the total population and $1.65 /$ 100,000 year for those older than 50 years.

Complications probably related to shunt placement were experienced by 4 of the 12 patients with shunts $(33 \%)$. One developed a pulmonary embolism days after the surgery, resulting in acute respiratory distress syndrome to which the patient eventually succumbed. A traumatic subdural hematoma requiring surgical drainage occurred 1 month after shunting in another patient. Two patients experienced epileptic seizures, one at 14 months and the other at 2 years after shunt placement.

Alternative or additional neurologic diagnoses were later given to 5 of the 12 patients with shunts (42\%). These included a clinical diagnosis of dementia with Lewy bodies in case 10 and multi-infarct dementia in case 11 . Electrophysiologically confirmed orthostatic myoclonus was subsequently identified as contributing to the gait problems of case 7 , and on subsequent postmortem examination, the patient was found to have argyrophilic grain disease. In case 5 , a second pathologically confirmed diagnosis of argyrophilic grain disease was noted in association with Braak and Braak stage IV, National Institute on Aging-Reagan intermediate likelihood Alzheimer pathology. Finally, postmortem examination of case 8 revealed progressive supranuclear palsy (PSP) neuropathology. None of the patients given an additional neurologic diagnosis after shunting experienced definite improvement in any symptom at 3 years.

DISCUSSION Although NPH is often high on the differential diagnosis for seniors with gait problems, our community-based data suggest that 1) clinical and neuroimaging features highly suggestive of NPH are relatively uncommon and 2) most patients originally suspected of having NPH do not respond to a CSF removal trial. The relatively low incidence of shunting for NPH approximates that of PSP in this county. ${ }^{4}$ Similarly, low incidence figures have been described in several European reports. ${ }^{5-9}$ Conversely, relatively high prevalence figures $(0.4 \%-14 \%)$ have been estimated when members of a population are screened for compatible clinical or neuroimaging features. ${ }^{10-14}$ Because these findings are nonspecific, studies that simply tabulate gait disturbance, dementia, incontinence, and ventriculomegaly without broader reference to the overall clinical context probably overestimate NPH prevalence. In contrast, our 
study captured only those thought to have NPH in the context of a thorough neurologic evaluation. It is possible some residents with NPH were initially managed by non-Mayo Clinic primary providers who then referred out of county, causing our incidence rate to be falsely low. However, given our community's high index of suspicion for this diagnosis and its usual referral patterns including residents' ready access to neurologists, we believe our ascertainment is as ideal as possible.

In our series, initial responses to ventriculoperitoneal shunting were quite promising although usually were not sustained. Objective measures were not uniformly available; thus, our study is limited by the retrospective and somewhat subjective nature of outcome determination. However, because all parties were significantly invested in the procedure and hoped for a benefit, we think it unlikely that the documented observations of the patients, family members, and treating clinicians significantly underestimate efficacy. To our knowledge, the present study represents the only community-based series in which long-term outcomes are addressed. Three- to 5 -year outcomes have been reported in several referral-based series which, in keeping with our observations, generally report initial response rates greater than $70 \%$. Some series report significant decline among initial responders, ${ }^{2,15-18}$ whereas others have shown that improvements are largely sustained over time. ${ }^{19-23}$ For several of these series, the loss to follow-up was substantial. Patients whose mobility deteriorates or who require placement in extended care facilities may be less able to return to their original surgical center, introducing a substantial source of bias. In contrast, we were able to assess outcomes among this subset.

Nearly half of our patients were later given an alternative neurologic diagnosis, and none of these realized sustained definite improvements after shunting. Whether they truly had NPH before developing a second neurologic disease is difficult to determine. However, it seems likely that in some, in particular the patient with pathologically proven PSP, the laterdiagnosed disorder had been responsible for the entire clinical picture. All 3 patients undergoing brain autopsy had neuropathologic findings of neurodegenerative disease, consistent with observations that more than two-thirds of patients receiving shunts for $\mathrm{NPH}$ have one or more pathologic markers of Alzheimer disease, and those with a moderate to severe Alzheimer disease burden are significantly less likely to respond to shunting. ${ }^{24}$ However, a favorable early response does not exclude an underlying neurodegenerative condition, as evidenced by our patient with PSP who had early, albeit transient, improve- ments after shunting. It is clear that careful diagnostic testing for alternative diagnoses is advisable before shunting; because newer techniques are able to diagnose neurodegenerative disease at earlier stages, the overall response to shunting may improve simply because of a shrinking denominator.

It is noteworthy that no patient with moderate to severe postural instability showed long-term definite improvement after shunting, and 4 of these 5 (excluding the patient who died perioperatively) were later given an additional neurologic diagnosis. Earlier reports have also suggested that imbalance is not likely to respond to CSF removal. ${ }^{25,26}$ It may be that this symptom is a hallmark of underlying neurodegenerative disease. Because postural instability is quite disabling, it is often the symptom initially prompting evaluation; our experience suggests that shunting is probably not the best course of action for these patients.

Patients and their families often perceive shunting to be a simple, safe procedure. However, it is not without risk. In our series, 1 patient experienced perioperative death and 3 more developed complications precipitated by the procedure. The one-third of our patients ( 4 of 12) with complications is in keeping with the $38 \%$ complication rate reported in a systematic review of prior series. ${ }^{16}$ Given that improvements after shunting for suspected NPH are often short-lived and given the significant potential for shunt-related complications, clinicians advising these patients need to remain mindful of the diagnostic limitations and maintain a reasonable perspective on the potential benefits of invasive CSF diversion.

\section{AUTHOR CONTRIBUTIONS}

Dr. Klassen: drafting/revising the manuscript, study concept or design, analysis or interpretation of data, acquisition of data, and statistical analysis. Dr. Ahlskog: drafting/revising the manuscript, study concept or design, analysis or interpretation of data, and study supervision.

\section{DISCLOSURE}

Dr. Klassen reports no disclosures. Dr. Ahlskog received the Fred Springer Award from the American Parkinson's Disease Association; serves on the editorial boards of Parkinsonism and Related Disorders and Clinical Neuropharmacology; receives royalties from the publication of The Parkinson's Disease Treatment Book (Oxford University Press, 2005), Parkinson's Disease Treatment Guide for Physicians (Oxford University Press, 2009), Parkinson's Disease and Movement Disorders (Humana Press, 2000), and Surgical Treatment of Parkinson's Disease and Other Movement Disorders (Humana Press, 2003); and receives research support from NIH/NINDS.

Received January 7, 2011. Accepted in final form April 20, 2011.

\section{REFERENCES}

1. Adams RD, Fisher CM, Hakim S, Ojemann RG, Sweet WH. Symptomatic occult hydrocephalus with "normal" cerebrospinal-fluid pressure: a treatable syndrome. N Engl J Med 1965;273:117-126. 
2. Petersen RC, Mokri B, Laws ER Jr. Surgical treatment of idiopathic hydrocephalus in elderly patients. Neurology 1985;35:307-311.

3. Marmarou A, Bergsneider M, Klinge P, Relkin N, Black PM. The value of supplemental prognostic tests for the preoperative assessment of idiopathic normal-pressure hydrocephalus. Neurosurgery 2005;57:S17-S28.

4. Bower JH, Maraganore DM, McDonnell SK, Rocca WA. Incidence of progressive supranuclear palsy and multiple system atrophy in Olmsted County, Minnesota, 1976 to 1990. Neurology 1997;49:1284-1288.

5. Brean A, Eide PK. Prevalence of probable idiopathic normal pressure hydrocephalus in a Norwegian population. Acta Neurol Scand 2008;118:48-53.

6. Brean A, Fredo HL, Sollid S, Muller T, Sundstrom T, Eide PK. Five-year incidence of surgery for idiopathic normal pressure hydrocephalus in Norway. Acta Neurol Scand 2009;120:314-316.

7. Tisell M, Hoglund M, Wikkelso C. National and regional incidence of surgery for adult hydrocephalus in Sweden. Acta Neurol Scand 2005;112:72-75.

8. Krauss JK, Halve B. Normal pressure hydrocephalus: survey on contemporary diagnostic algorithms and therapeutic decision-making in clinical practice. Acta Neurochir 2004; 146:379-388.

9. Vanneste J, Augustijn P, Dirven C, Tan WF, Goedhart ZD. Shunting normal-pressure hydrocephalus: do the benefits outweigh the risks? A multicenter study and literature review. Neurology 1992;42:54-59.

10. Marmarou A, Young HF, Aygok GA. Estimated incidence of normal pressure hydrocephalus and shunt outcome in patients residing in assisted-living and extended-care facilities. Neurosurg Focus 2007;22:E1.

11. Trenkwalder C, Schwarz J, Gebhard J, et al. Starnberg trial on epidemiology of Parkinsonism and hypertension in the elderly: prevalence of Parkinson's disease and related disorders assessed by a door-to-door survey of inhabitants older than 65 years. Arch Neurol 1995;52:1017-1022.

12. Hiraoka K, Meguro K, Mori E. Prevalence of idiopathic normal-pressure hydrocephalus in the elderly population of a Japanese rural community. Neurol Med Chir 2008; 48:197-199.

13. Iseki C, Kawanami T, Nagasawa $\mathrm{H}$, et al. Asymptomatic ventriculomegaly with features of idiopathic normal pressure hydrocephalus on MRI (AVIM) in the elderly: a prospective study in a Japanese population. J Neurol Sci 2009; 277:54-57.
14. Tanaka N, Yamaguchi S, Ishikawa H, Ishii H, Meguro K. Prevalence of possible idiopathic normal-pressure hydrocephalus in Japan: the Osaki-Tajiri project. Neuroepidemiology 2009;32:171-175.

15. Malm J, Kristensen B, Stegmayr B, Fagerlund M, Koskinen LO. Three-year survival and functional outcome of patients with idiopathic adult hydrocephalus syndrome. Neurology 2000;55:576-578.

16. Hebb AO, Cusimano MD. Idiopathic normal pressure hydrocephalus: a systematic review of diagnosis and outcome. Neurosurgery 2001;49:1166-1184.

17. Savolainen S, Hurskainen H, Paljarvi L, Alafuzoff I, Vapalahti M. Five-year outcome of normal pressure hydrocephalus with or without a shunt: predictive value of the clinical signs, neuropsychological evaluation and infusion test. Acta Neurochir 2002;144:515-523.

18. Kahlon B, Sjunnesson J, Rehncrona S. Long-term outcome in patients with suspected normal pressure hydrocephalus. Neurosurgery 2007;60:327-332.

19. Raftopoulos C, Massager N, Baleriaux D, Deleval J, Clarysse S, Brotchi J. Prospective analysis by computed tomography and long-term outcome of 23 adult patients with chronic idiopathic hydrocephalus. Neurosurgery 1996;38:51-59.

20. Mori K. Management of idiopathic normal-pressure hydrocephalus: a multiinstitutional study conducted in Japan. J Neurosurg 2001;95:970-973.

21. Aygok G, Marmarou A, Young HF. Three-year outcome of shunted idiopathic NPH patients. Acta Neurochir Suppl 2005;95:241-245.

22. Tisell M, Hellstrom P, Ahl-Borjesson G, et al. Long-term outcome in 109 adult patients operated on for hydrocephalus. Br J Neurosurg 2006;20:214-221.

23. Pujari S, Kharkar S, Metellus P, Shuck J, Williams MA, Rigamonti D. Normal pressure hydrocephalus: long-term outcome after shunt surgery. J Neurol Neurosurg Psychiatry 2008;79:1282-1286.

24. Hamilton R, Patel S, Lee EB, et al. Lack of shunt response in iNPH with AD pathology. Ann Neurol 2010;68:535540.

25. Stolze H, Kuhtz-Buschbeck JP, Drucke H, et al. Gait analysis in idiopathic normal pressure hydrocephalus: which parameters respond to the CSF tap test? Clin Neurophysiol 2000;111:1678-1686.

26. Bugalho P, Guimaraes J. Gait disturbance in normal pressure hydrocephalus: a clinical study. Parkinsonism Relat Disord 2007;13:434-437.

\section{Neurology ${ }^{\circledR}$ Launches Subspecialty Alerts by E-mail!}

Customize your online journal experience by signing up for e-mail alerts related to your subspecialty or area of interest. Access this free service by visiting http://www.neurology.org/site/subscriptions/ etoc.xhtml or click on the "E-mail Alerts" link on the home page. An extensive list of subspecialties, methods, and study design choices will be available for you to choose from-allowing you priority alerts to cutting-edge research in your field! 


\section{Neurology}

\section{Normal pressure hydrocephalus: How often does the diagnosis hold water? Bryan T. Klassen and J. Eric Ahlskog \\ Neurology 2011;77;1119-1125 Published Online before print August 17, 2011 \\ DOI 10.1212/WNL.0b013e31822f02f5}

\section{This information is current as of August 17, 2011}

\section{Updated Information \& Services}

Supplementary Material

\section{References}

\section{Citations}

Subspecialty Collections

Permissions \& Licensing

Reprints including high resolution figures, can be found at: http://n.neurology.org/content/77/12/1119.full

Supplementary material can be found at: http://n.neurology.org/content/supp1/2011/09/16/WNL.0b013e31822f0 2f5.DC1

This article cites 26 articles, 5 of which you can access for free at: http://n.neurology.org/content/77/12/1119.full\#ref-list-1

This article has been cited by 6 HighWire-hosted articles: http://n.neurology.org/content/77/12/1119.full\#\#otherarticles

This article, along with others on similar topics, appears in the following collection(s):

All Cognitive Disorders/Dementia

http://n.neurology.org/cgi/collection/all_cognitive_disorders_dementia Gait disorders/ataxia

http://n.neurology.org/cgi/collection/gait_disorders_ataxia Hydrocephalus

http://n.neurology.org/cgi/collection/hydrocephalus

Incidence studies

http://n.neurology.org/cgi/collection/incidence_studies

Information about reproducing this article in parts (figures,tables) or in its entirety can be found online at:

http://www.neurology.org/about/about_the_journal\#permissions

Information about ordering reprints can be found online:

http://n.neurology.org/subscribers/advertise

Neurology ${ }^{\circledR}$ is the official journal of the American Academy of Neurology. Published continuously since 1951, it is now a weekly with 48 issues per year. Copyright Copyright $@ 2011$ by AAN Enterprises, Inc.. All rights reserved. Print ISSN: 0028-3878. Online ISSN: 1526-632X.

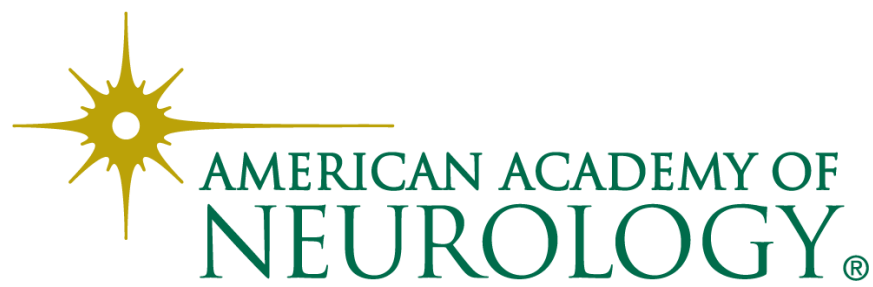

